\title{
Synthesis of Highly Mono-dispersed AgNPs within PVP Matrix via Gamma Irradiation
}

\author{
Z. I. Ali, H. H. Saleh and T. A. Afify
}

Radiation Chemistry Dept., National Centre for Radiation Research and Technology (NCRRT), B. O. Box; 29 Nasr City, Egypt.

\begin{abstract}
GAMMA-RADIATION synthesized silver/poly(vinyl pyrrolidone) (Ag/PVP) nanocomposite films were prepared using PVP as a stabilizer. The prepared films were characterized by Ultra Violet/ Visible (UV/vis) spectroscopy, Transmission Electron Microscopy (TEM), X-ray Diffraction (XRD) and Fourier Transform Infera Red (FTIR). The UV/vis analysis showed the appearance of surface plasmon resonance peaks at 403-406 nm confirming the formation of silver nanoparticles (AgNPs). The TEM images confirmed the formation of a highly mono-disperse and narrow size distributed AgNPs and the particle size was increased by increasing $\mathrm{Ag}^{+}$ion molar concentration. The XRD patterns exhibit four diffraction peaks revealed that the AgNPs are formed within PVP matrix and its crystal structure is face centre cubic (fcc) structure. The calculated values of crystalline particle size $(\mathrm{D}, \mathrm{nm})$ showed that both $\mathrm{AgNO}_{3}$ molar concentration and irradiation dose are determining factors in controlling the particle size of AgNPs. FTIR spectra illustrate that the formation Ag/ PVP nano-composites leads to change in peak position, peak shape and peak intensity of the characteristic peaks. The differences in the Ag/PVP nanocomposite spectrum compared to that of pure PVP indicated the formation of coordination bonding between nitrogen and AgNPs, as well as between oxygen and AgNPs.

Keywords: AgNPs, PVP, UV/ VIS spectroscopy, TEM, XRD $\gamma$-rays.
\end{abstract}

Metal nano-structures with functional properties have been extensively studied recently, since they play an important role in many diverse applications. Among the various metal nano-structures, noble metal nanoparticles have attracted considerable attention, due to their unusual optical and chemical properties. Recently, lot of researches have been focused on AgNPs because of their important applications especially in nano-electronics, magnetic and biosensors, data storage, catalysis. Water soluble AgNPs/polymer composites were found to possess bioactive properties to suppress bacteria's growth. Most of these 
applications require small sized nano-particles with a narrow size distribution. Such properties and applications strongly depend on the morphologies and dimensions of the silver nano-structures. The synthesis of AgNPs with wellcontrolled morphology and a narrow size distribution is important for uncovering their properties and for achieving their practical applications (Puiso et al., 2011).

Polymers have been frequently used as particle stabilizer and capping agent in irradiation synthesis of metal nanoparticles, since they prevent agglomeration and precipitation of the nanoparticles. The embedding of such nanoparticles in polymer matrix is also advantageous from point of view of film casting. PVP is known to be a good stabilizer for metal nanoparticles. Moreover, it is a water soluble polymer, so it can react with metal salt via formation of chelae with metal cations in an aqueous solution and also act as a capping agent. Also, PVP is a potential material with very high dielectric strength; furthermore, it has a good charge capacity and dopant-dependent optical properties. The preparation of inorganic/organic material is believed to remarkably improve a wide range of properties of the polymers due to nano-sized dispersion. Among these properties, the enhancement of optical, structural and thermal property is one that has been observed in many polymer nano-composites (Basavaraja et al., 2008).

Gamma irradiation offers many advantages for the preparation of metal nanoparticles. Large number of hydrated electrons $\left(\mathrm{e}_{\mathrm{aq}}^{-}\right)$produced during $\gamma$ irradiation can reduce the metal ions to zero valiant metal particles. A summation made by many authors suggested that irradiation cause a chemical change in polymers, including cross linking, chain scission, formation of alkynes groups, and depletion of hetero atoms (e.g. N, S or O). In recent years studies on the optical and electrical properties of polymers have attracted much attention in view of their application in optical devices. The optical properties of polymers can be suitably modified by the addition of dopants depending on their reactivity with the host matrix. Since $\mathrm{AgNO}_{3}$ is a fast conducting ion in a number of crystalline and amorphous materials, its incorporation within a polymeric system may be expected to enhance its electrical and optical performance (Virk et al., 2001).

\section{Materials and Methods}

Silver nitrate $\left(\mathrm{AgNO}_{3}\right)$ and Isopropyl alcohol were obtained from Nice Chemicals PVT, LTD, India. PVP, M.wt $\approx 40,000 \mathrm{~g} / \mathrm{mol}$ in the powder form 
was obtained from Sigma, USA. All chemicals were used without further purification.

\section{Preparation of Ag/PVP nanocomposite films}

Firstly, PVP solution was prepared by dissolving $4.5 \mathrm{~g}$ PVP in $140 \mathrm{ml}$ of de-ionized water and $10 \%$ of isopropyl alcohol was added to the PVP solution with stirring. After that, $10 \mathrm{ml}$ of freshly prepared $\mathrm{AgNO}_{3}$ solution $(0.001$ and $0.005 \mathrm{M})$ were added to the above PVP solution. Finally, the solution was placed into test tubes and irradiated with different irradiation doses (50-150 kGy) using Co-60 $\gamma$-cell-220 source (manufactured by the Atomic Energy Authority of India with $3.48422 \mathrm{kGy} / \mathrm{h}$ dose rate) installed at NCRRT, Nasr City, Egypt, and then casted to be a film after irradiation process.

\section{Characterization techniques}

\section{UV/vis spectroscopy}

UV/VIS spectrophotometer (Unicam, England) was used for scanning the absorption spectra in the range from $190 \mathrm{~nm}$ to $1000 \mathrm{~nm}$ wavelengths and measuring the optical density at $\lambda_{\max }$ for non-irradiated and irradiated samples.

\section{Transmission electron microscopy (TEM)}

TEM observation was performed with JEOL JSM-100 CX, Shimadzu Co. electron microscope operated at an acceleration voltage of $80 \mathrm{KV}$. The sample for TEM analysis were prepared by deposition of a single drop of the Ag/PVP nanocomposite dispersion on a 300 mech copper grid with a carbon film over a filter paper which absorbed excess solution. The copper grid was allowed to dry at room temperature.

\section{$X$-ray diffraction $(X R D)$}

XRD patterns of the investigated samples were determined by X-ray diffractometer (a Shimadzu XRD 600). XRD patterns in the $\theta$ range between $2 \theta$ of $4^{\circ}$ to $70^{\circ}$ were obtained at a scan rate of $2^{\circ} / \mathrm{min}$ on the diffract meter with $\mathrm{CuK}$ radiation source, a generator voltage of $40 \mathrm{KV}$, a generator current of $40 \mathrm{~mA}$ and a wavelength of $0.1546 \mathrm{~nm}$ at room temperature. All the diffraction patterns were examined at room temperature and under constant operation conditions. 


\section{Fourier Transform Infrared (FTIR) Spectroscopy}

An ATI Mattson (Genesis series, Unicam, England) FTIR spectroscopy was used to scan and measure the absorption spectra at a resolution of $4 \mathrm{~cm}^{-1}$. The spectra of non-irradiated and irradiated samples were measured before and directly after irradiation.

\section{Results and Discussion}

\section{UV/ Vis spectroscopic study of highly mono-dispersed AgNPs}

When noble metal nanoparticles are excited by electromagnetic radiation, they exhibit collective oscillations of their conduction electrons that result in both absorption and scattering of the incident radiation. The excitation spectrum, which is the sum of the absorption and scattering spectra, of such nanoparticles in a solution, often, measured using UV/vis spectrophotometer. In a solution, of nearly mono dispersed spherical gold or AgNPs in solution, the extinction spectrum exhibits a single peak known as the localized surface plasmon resonance (LSPR). The ability to manipulate and predict the LSPR of metal nanoparticle systems is desirable in several technological applications (Malinsky et al., 2001).

The interaction of ionizing radiation such as $\gamma$-ray with aqueous solution is well established. As water is the major component of aqueous solution, most part of energy of the radiation is absorbed in water, which causes radiolysis of water. The radiolysis transient species, namely hydrated electron $\left(\mathrm{e}_{\mathrm{aq}}\right)$, hydrogen atom radical $\left(\mathrm{H}^{\circ}\right)$ and hydroxyl radical $\left({ }^{\circ} \mathrm{OH}\right)$, which they are highly reactive in nature. The first two radicals can reduce many types of metal ions to lower valences and metal atoms. Finally these metal atoms coalesce to form metal nanoparticles in presence of a capping agent such as polymers, legends, surfactants etc. The ${ }^{\circ} \mathrm{OH}$ radical being oxidizing in nature and it can oxidized back the metal atoms. Hence to scavenge ${ }^{\circ} \mathrm{OH}$ radical, isopropyl alcohol is added to the system. The reaction product of isopropyl alcohol with ${ }^{\circ} \mathrm{OH}$ radical is propyl $\left(\left(\mathrm{CH}_{3}\right)_{2} \mathrm{CH}^{*}\right)$ radical. There is one more advantage of using isopropyl alcohol; the isopropyl radical produced is a reducing agent and it is capable of reducing metal ions like $\mathrm{Ag}^{+}$ion (Misral et al., 2012).

The characterization of the prepared Ag/PVP nanocomposite using $\gamma$-rays was presented in Fig. 1. showed that the formation of AgNPs via $\gamma$-irradiation through the observed surface plasmon peak (SPP) at about 403-406 nm. The non- 
irradiated Ag/ PVP sample I, does not exhibit any peak through the entire wavelength range. On the other hand, the irradiated Ag/ PVP samples showed a surface SPP in the range 403-406 nm. Also, one can notice that there is a little variation in the SPP intensity due to irradiation as can be attributed to the lower molar concentration of $\mathrm{AgNO}_{3}(0.001 \mathrm{M})$. The surface plasmon phenomenon, i.e., cloud electromagnetic waves coupled with conduction band (CB) electrons, shows the peak at $\approx(406 \mathrm{~nm})$, shifting to lower wavelength (blue shift), with increasing irradiation dose from 25 to $100 \mathrm{kGy}$ indicating a decrease of particle size of AgNPs within PVP matrix.

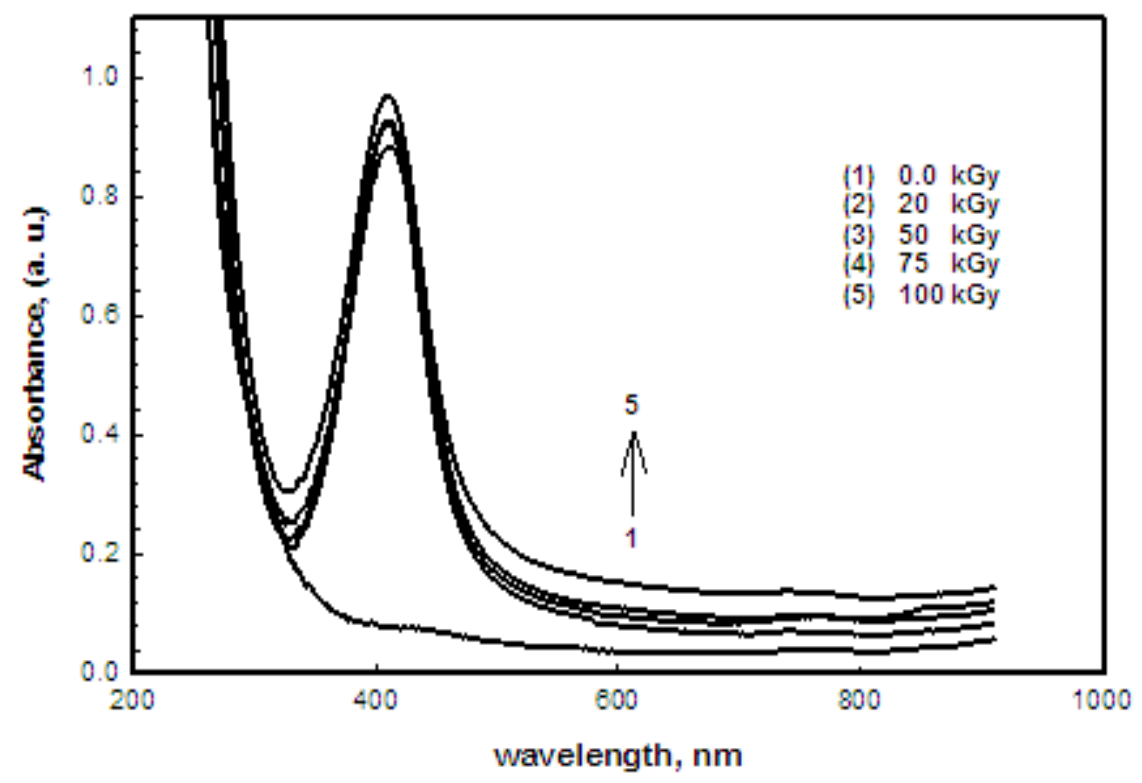

Fig. 1. UV/VIS spectra of non-irradiated and irradiated Ag /PVP nanocomposite films $\left(0.001 \mathrm{M} \mathrm{AgNO}_{3}\right)$.

Fig. 2. shows the UV/vis spectra of non-irradiated and irradiated Ag/PVP nanocomposite films containing higher molar concentration of $\mathrm{AgNO}_{3}(0.005$ M). The characteristic peak of the AgNPs appeared in the range of $414 \mathrm{~nm}$ to $410 \mathrm{~nm}$, with noticeable variation in the peak shape and intensity depending on the value of the absorbed irradiation dose. The intensities of the SPR absorption of the AgNPs gradually increased with increasing either irradiation dose or $\mathrm{Ag}^{+}$ molar concentration, indicating that more AgNPs were generated. Also, the variation in the intensity at $\lambda_{\max }$ is more pronounced at higher $\mathrm{AgNO}_{3}$ molar 
concentration. The slight red shift of the peak maximum to higher wavelengths implies that the average sizes of the AgNPs slightly increase with increasing $\mathrm{Ag}^{+}$molar concentration, (Fig. 1, 2).

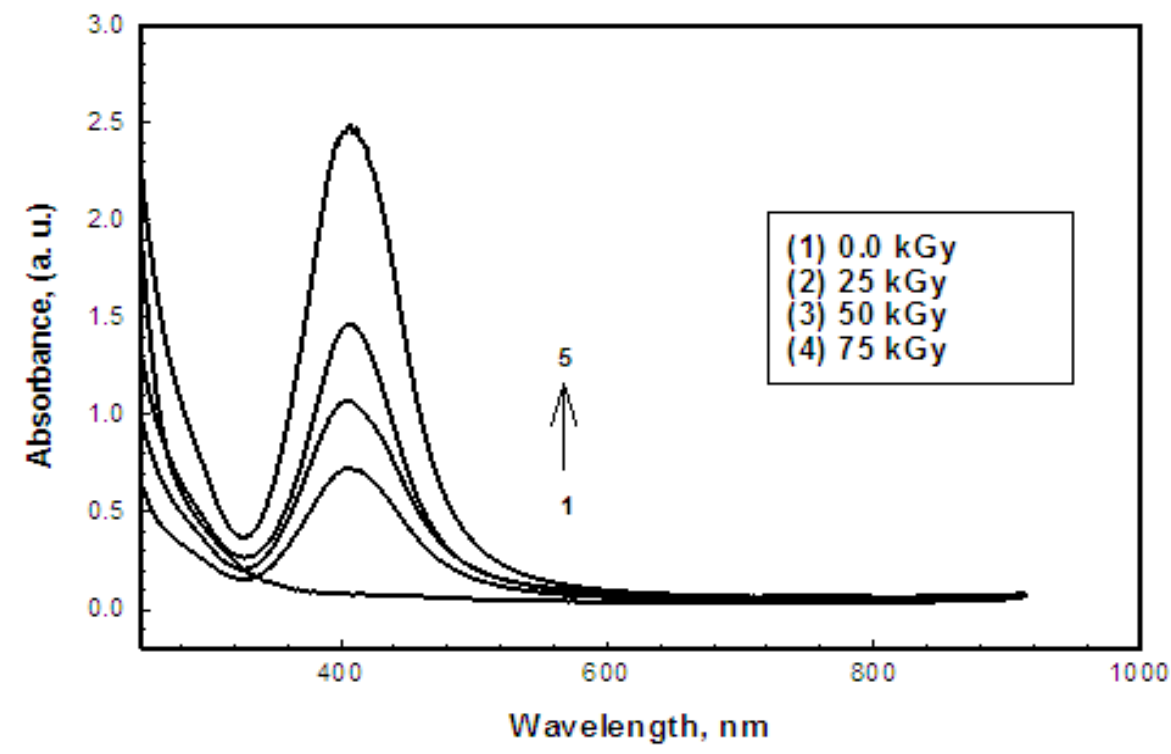

Fig. 2. UV/vis spectra of non-irradiated and irradiated Ag/PVP nanocomposite films (0.005 M AgNO3).

The correlation between the AgNPs size and $\mathrm{AgNO}_{3}$ concentration was reported to depend on the following three different situations as described by Chen and $\mathrm{Wu}(2000)$. The first, if the number of Ag metal nuclei increased faster than that of total ions, smaller particles would be obtained. The second, if the increase of metal nucleus number was proportional to that of total metal ion number, the particle size might remain unchanged. The third, when the number of metal nuclei remained constant or increased slower than that of total ions, the particle size would become larger with the increase of metal ion concentration. Based on the above observation, our case appears to belong to the third situation. According to this correlation, AgNPs can be efficiently prepared with controlled size. Furthermore, the surface SPP become broader with increasing $\mathrm{AgNO}_{3}$ molar concentration. This indicates that, the symmetric and narrow absorption peak implies the narrow size distribution of the AgNPs at lower $\mathrm{Ag}^{+}$ion concentration.

Assuming that AgNPs have spherical shape, Mie theory (Puiso et al., 2008) was applied for the evaluation of AgNPs size in $\mathrm{Ag} / \mathrm{PVP}$ nanocomposites. 
Correlation between SPR peak position and particle size experimentally established by Evanoff and Chumnov (2004) and Wilson et al. (2007) was used for the calculation of silver nanoparticle size in polymer matrix using experimental data: $\quad \mathbf{D}=\mathbf{0 . 7 1 5} \lambda_{\max }-\mathbf{2 5 8}$

where, D is the particle diameter (nm), and $\lambda_{\max }$ is the SPR peak position in UV/vis absorbance spectrum. The calculated AgNPs particle size was listed in Table 1. The correlation between the irradiation dose and the observed wavelength at maximum absorption toward the determined particle size are reported in Table 1. It can be clearly seen that both particle size and the absorbance at maximum of surface SPP and the corresponding particle size decrease with increasing irradiation dose. In addition, the level of $\lambda_{\max }$ and particle size of AgNPs in PVP matrix is being to be of higher values with increasing the molar concentration of $\mathrm{AgNO}_{3}$. In this respect, one can conclude that the concentration of $\mathrm{AgNO}_{3}$ may be one of the important parameter affecting the physico-chemical and structural properties of the prepared Ag/PVP nanocomposite films. Also, the concentration of $\mathrm{AgNO}_{3}$ may be decides the ultimately quantity of the metal nanoparticles in PVP matrix and also affect the size diameter, size distribution and type of nanoparticles.

TABLE 1. The estimated absorbance at $\lambda_{\max }$, and particle size from UV/VIS spectroscopic analysis.

\begin{tabular}{|c|c|c|c|}
\hline $\begin{array}{c}\mathbf{A g N O}_{3} \\
\text { concentration }\end{array}$ & Dose (kGy) & $\boldsymbol{\lambda}_{\max }(\mathbf{n m})$ & $\mathbf{D}(\mathbf{n m})$ \\
\hline \multirow{3}{*}{$0.005 \mathrm{M}$} & 25 & 414 & 38.0 \\
\cline { 2 - 4 } & 50 & 412 & 36.5 \\
\cline { 2 - 4 } & 75 & 410 & 35.0 \\
\cline { 2 - 4 } & 100 & 410 & 35.0 \\
\hline \multirow{3}{*}{$0.001 \mathrm{M}$} & 25 & 406 & 32.3 \\
\cline { 2 - 4 } & 50 & 405 & 31.5 \\
\cline { 2 - 4 } & 75 & 403 & 30.0 \\
\cline { 2 - 4 } & 100 & 403 & 30.0 \\
\hline
\end{tabular}

The role of PVP in the formation of AgNPs can be recognized as follow: The PVP is a homopolymer whose individual unit contains an amide group. The $\mathrm{N}$ and $\mathrm{O}$ atoms of this polar group probably have a strong affinity for the silver ions and metallic silver. PVP macromolecule in the matrix may take part in some form of association with the $\mathrm{Ag}^{+}$ion and reduces these ions into metallic nanoparticles which in turn increase the probability of nucleus formation. In 
addition the resultant metallic silver particles are faster capped by PVP macro molecule of higher $\mathrm{Ag}^{+}$ion concentration than by that of lower concentration. The PVP macro molecules are regarded as the stabilizing agents, which form a steric hindrance around the AgNPs to prevent them from aggregation greatly by electrostatic interactions (Xie et al., 2006).

\section{TEM of Ag/PVP nanocomposites}

In order to obtain information about the external shape, dimension and distribution of the prepared AgNPs embedded in PVP matrix, Fig. 3, 4 represent the TEM images of $100 \mathrm{kGy}$ irradiated $\mathrm{Ag} / \mathrm{PVP}$ nanocomposites containing 0.001 and $0.005 \mathrm{M} \mathrm{AgNO}_{3}$. It is found that at lower $\mathrm{Ag}^{+}$ion concentration; a number of well dispersed uniform size spherical nanoparticles can be clearly seen in the TEM image with fairly even size distribution and has an external spherical shape with average particle size $13 \mathrm{~nm}$ (Fig. 3). With increasing $\mathrm{Ag}^{+}$ ion concentration (i.e. increasing AgNPs content), the sample produced network consisting of $\mathrm{Ag}$ clusters separately distribution within the entire polymer matrix (Fig. 4).

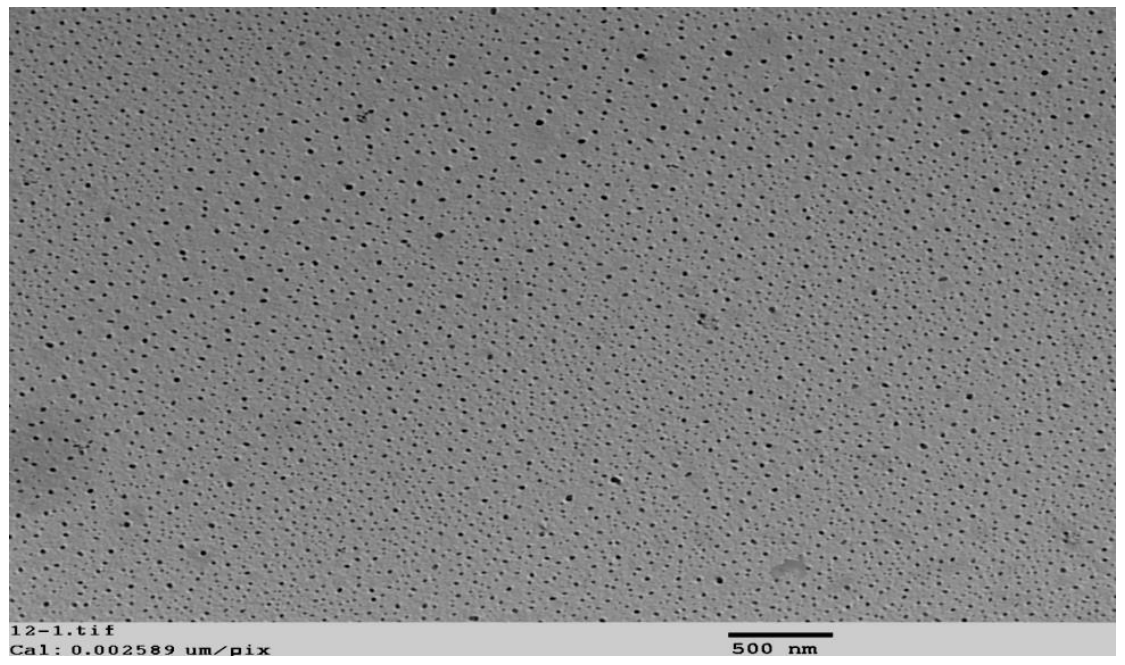

Fig.3. TEM image of $100 \mathrm{kGy}$ irradiated Ag/PVP nanocomposite containing $0.001 \mathrm{M} \mathrm{AgNO}_{3}$

In addition, the size of the observed AgNPs increases with increasing $\mathrm{Ag}^{+}$ ion concentration to the range $20-30 \mathrm{~nm}$, and further more yields a particle with 
larger size distribution. The obtained TEM images illustrate that, the AgNPs are not aggregate at lower $\mathrm{Ag}^{+}$concentration and are well distributed within the polymer matrix. The non-aggregation may be due to the formation of sterric coils of PVP chains around the AgNPs, which was added during their synthesis. This provides chemical stability to nanoparticles even after embedded into PVP matrix. For higher dopant concentration of Ag in PVP, the TEM images illustrate the increase in the particle size. Such increase at higher dopant Ag concentration generally occurs as a result of the self-reactivity of the nanoparticles to aggregate. Finally, at lower $\mathrm{Ag}^{+}$ion concentration, well dispersed AgNPs with narrow size distribution were observed indicating the formation of $\mathrm{Ag}$ quantum dots. At higher $\mathrm{Ag}^{+}$ion concentration, $\mathrm{Ag}$ nanosphere with larger size was formed. The size of nano-particles, size distribution, and uniform dispersity increases with increasing $\mathrm{Ag}^{+}$ion concentration (Mahendia et al., 2011).

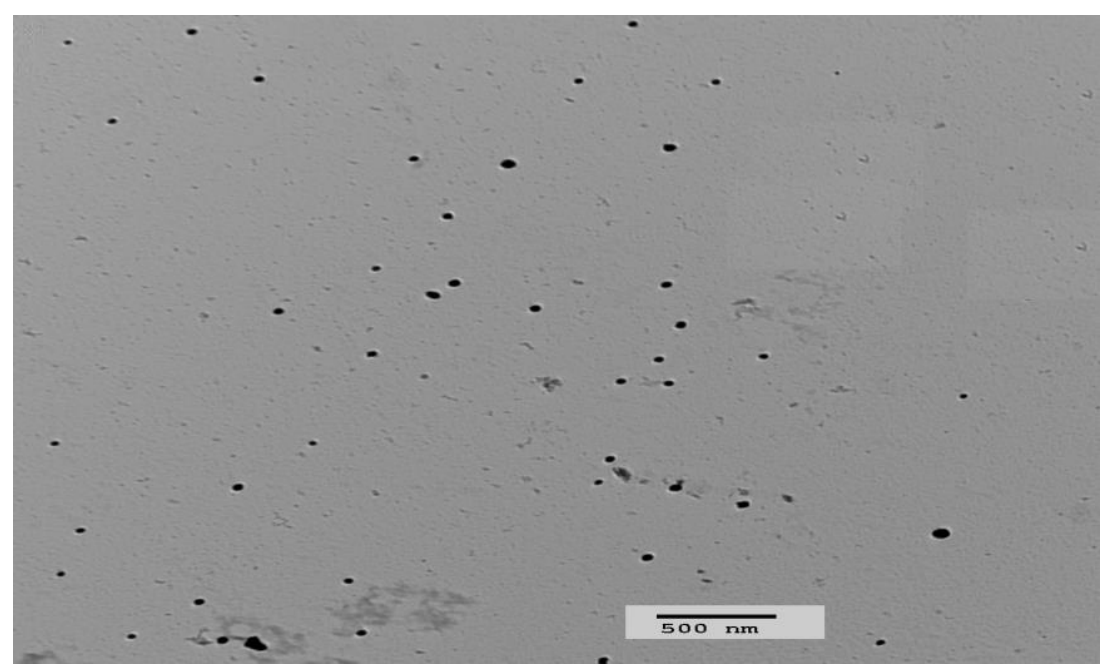

Fig. 4. TEM image of $100 \mathrm{kGy}$ irradiated Ag/PVP nanocomposite containing 0.005 $\mathrm{M} \mathrm{AgNO}{ }_{3}$.

\section{XRD characterization}

Fig. 5. displays the XRD patterns of irradiated Ag/PVP nanocomposites containing $0.005 \mathrm{M} \mathrm{AgNO}_{3}$. A more precise examination lead to two remarks: First, the irradiated nano-composites show five diffraction peaks at $2 \theta=22.1$, $37.9,43.2,63.7$ and $77.7^{\circ}$. These discernible peaks can be indexed to the planes 
(110), (111), (200), (220) and (311), respectively. The first amorphous scattering peak at $2 \theta=22.1^{\circ}$, this is the characteristic peak of PVP matrix. The other four peaks revealed that the AgNPs are formed in the PVP matrix and their crystal structure is fcc structure according to JCPDS (No.4-0783). This is a confirmation of the presence of AgNPs in PVP matrix as a result of irradiation. Second, as the irradiation dose increased from 50 to $100 \mathrm{kGy}$, the intensity of the $\mathrm{Ag}$ lines increases gradually in accordance with their small grain sizes (Fig. 5). Also, it can be seen that the prepared growth plane of the AgNPs is the (111) lattice plane which show the highest intensity all over the diffraction pattern. In addition, peaks at (200) and (220) reflection planes, corresponding to the high angle XRD pattern, supports the presence of AgNPs in the as-prepared nano-composites.

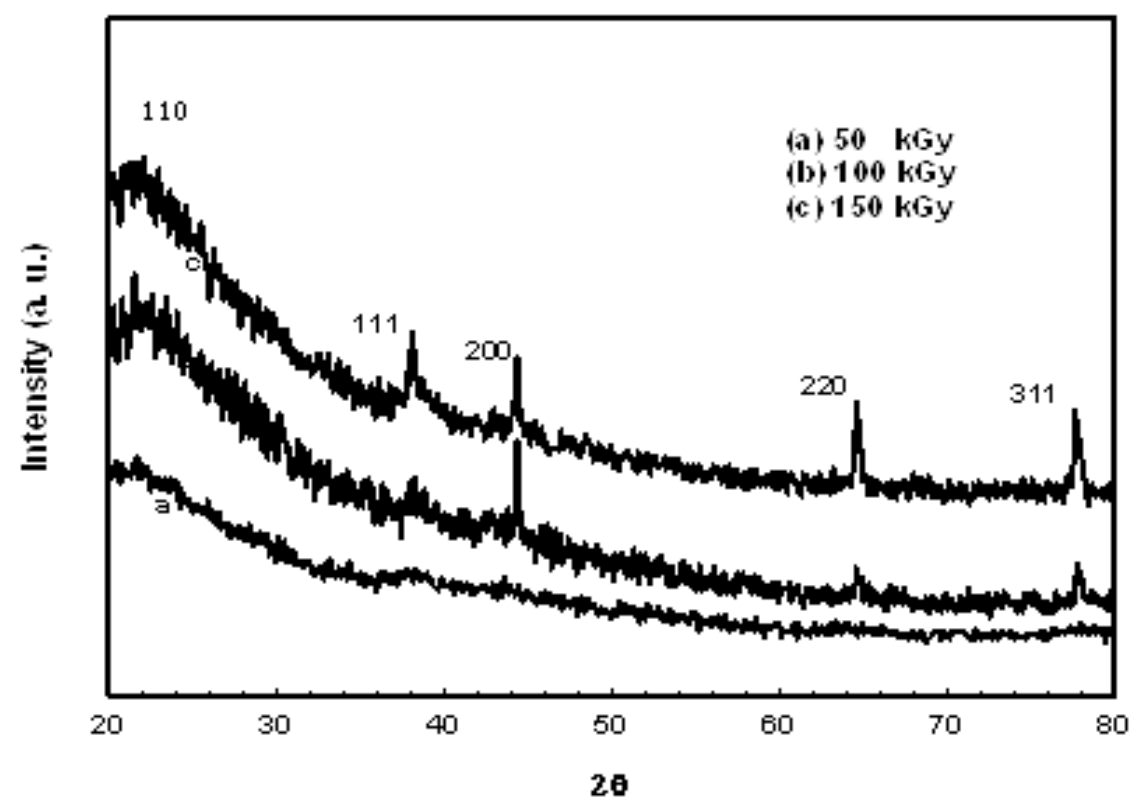

Fig. 5. XRD patterns of irradiated Ag/PVP nanocomposite (0.005 $\left.\mathrm{M} \mathrm{AgNO}_{3}\right)$.

\section{FTIR characterization}

The FTIR spectrum of PVP exhibited the following absorption bands: broad absorption band peaking at $3441 \mathrm{~cm}^{-1}$, corresponding to -OH group vibrations, followed by a peak at $2953 \mathrm{~cm}^{-1}$, assigned to vibration of the $-\mathrm{CH}$ group. The main feature of the PVP spectrum, whose monomer, N-vinyl-2pyrrolidone, contains an amide carbonyl group, is a strong band at $1660 \mathrm{~cm}^{-1}$. 
Other bands in this spectrum are observed at $1373 \mathrm{~cm}^{-1}$ and $1288 \mathrm{~cm}^{-1}$, due to the bond vibrations of the $\mathrm{NO}_{3}{ }^{-}$group and of the $\mathrm{N}-\mathrm{OH}$ complex, respectively. These results are in accordance with the results for PVP reported in literature (Wang et al., 2005 and Zhang et al., 1996).

Fig. 6. shows the FTIR spectra of irradiated Ag/PVP nanocomposite $\left(0.001 \mathrm{M} \mathrm{AgNO}_{3}\right)$. There are few differences in peak positions compared to the pure PVP, suggesting the bonding between PVP molecules and AgNPs. One of the main differences observed is the shift of the amide carbonyl group band of PVP from $1660 \mathrm{~cm}^{-1}$ toward $1665 \mathrm{~cm}^{-1}$ in the Ag/PVP nano-composite spectrum. Also, the positions of the peaks for tertiary nitrogen vibrations $(\approx$ $\left.1462 \mathrm{~cm}^{-1}\right), \mathrm{NO}_{3}^{-}$group vibrations $\left(\approx 1373 \mathrm{~cm}^{-1}\right)$, and the N-OH complex $(\approx$ $1288 \mathrm{~cm}^{-1}$ ), observed in the spectrum of PVP, were shifted to higher wavenumber, in the Ag/PVP nanocomposite spectrum, suggesting the coordination between nitrogen atom from PVP and AgNPs. All of the differences exhibited in the Ag/PVP nanocomposite spectrum compared to the spectrum of pure PVP indicated the coordination bonding between nitrogen and AgNPs, as well as between oxygen and AgNPs, most likely through the reactions shown at scheme 1 (Kan et al., 2010 and Zhang et al., 1996).

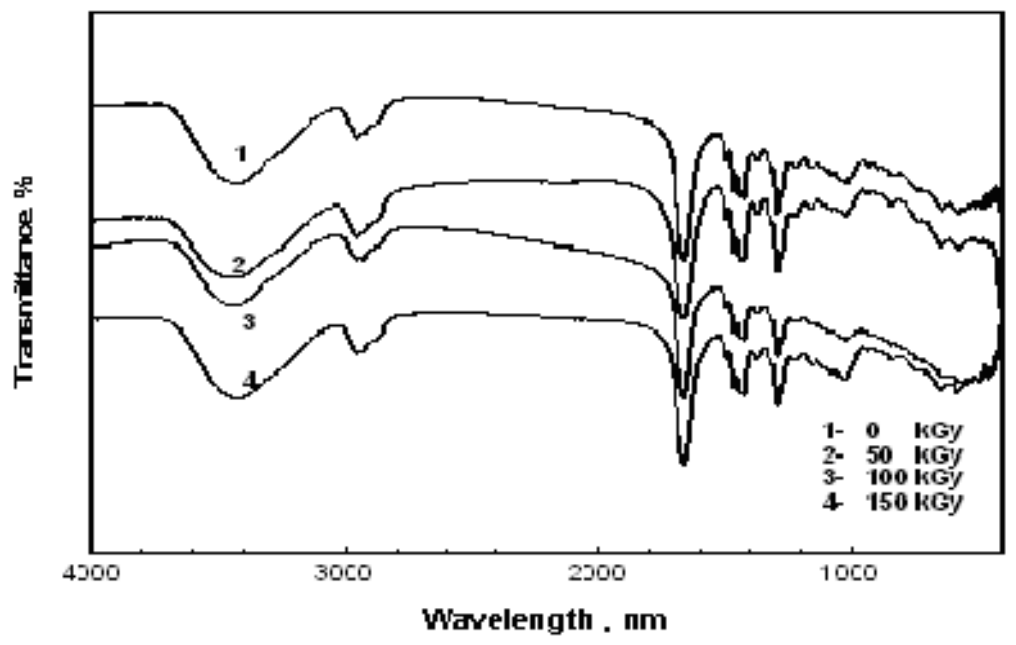

Fig. 6. FTIR spectra of irradiated Ag/PVP nanocomposites ( $\left(0.001 \mathrm{M} \mathrm{AgNO}_{3}\right)$.

Fig. 7. illustrate that the formation of Ag/PVP nanocomposite leads to the change in peak position, peak shape and peak intensity due to incorporation of 
various content of $\mathrm{Ag}^{+}$ion. One can notice that with increasing $\mathrm{Ag}^{+}$ion molar concentration, there are an observed shifted to lower wave number, and increase in the peak intensity for Ag/PVP nano-composite which suggesting the coordination between PVP and AgNPs. On the other hand, the broadening behaviour for some characteristic bands especially at $3400 \mathrm{~cm}^{-1}$ increases as a result of increasing $\mathrm{Ag}$ content in $\mathrm{Ag} / \mathrm{PVP}$ nanocomposite. These results suggest that the nitrogen atom is the important active site for the coordination of AgNPs.

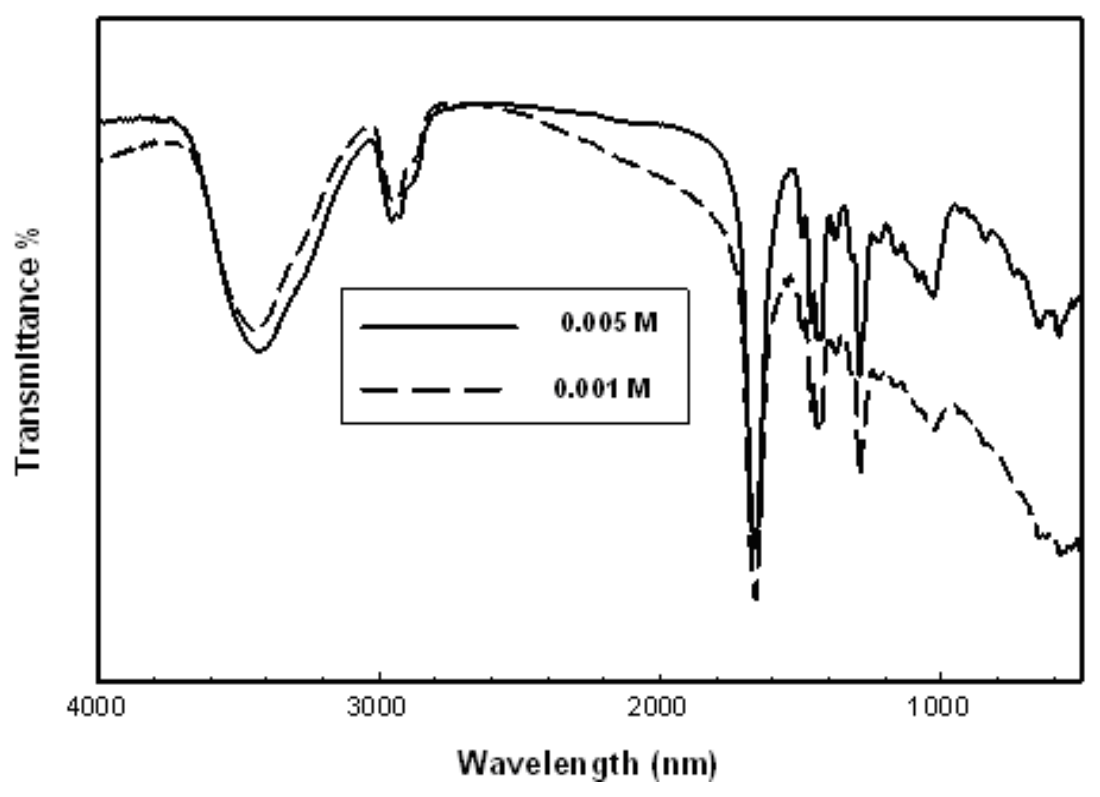

Fig.7. FTIR spectra of $100 \mathrm{kGy}$ irradiated Ag/PVP nanocomposites.

However, since all of the changes of the absorption band positions include nitrogen, it can be presumed that the main way of PVP to stabilize AgNPs in this radiolytically synthesized system, was trough the nitrogen in PVP that coordinated with AgNPs. It was reported previously that the size of AgNPs influenced the type of coordination bonding, due to the steric effect of the pyrrolidone ring as shown in scheme 1 (Wang et al., 2005). On the other hand, the electronegativity of nitrogen, lower than that of oxygen, results in the enhanced capability of electron donating of the nitrogen with respect to oxygen. This is the reason why the particles with the diameter smaller than $50 \mathrm{~nm}$ coordinated with nitrogen, regardless the steric effect. Therefore, PVP 
molecules can serve as protective molecules for AgNPs gamma-irradiation synthesis, because the nitrogen atoms, preferentially, but also oxygen atoms in the polar groups of the PVP repeated unit, $\mathrm{N}$-vinyl-2-pyrrolidone, can donate an electron pair to the sp hybridized Ag $\mathrm{p}$ ions, forming a linear coordinative bond (Jiang et al., 2004).
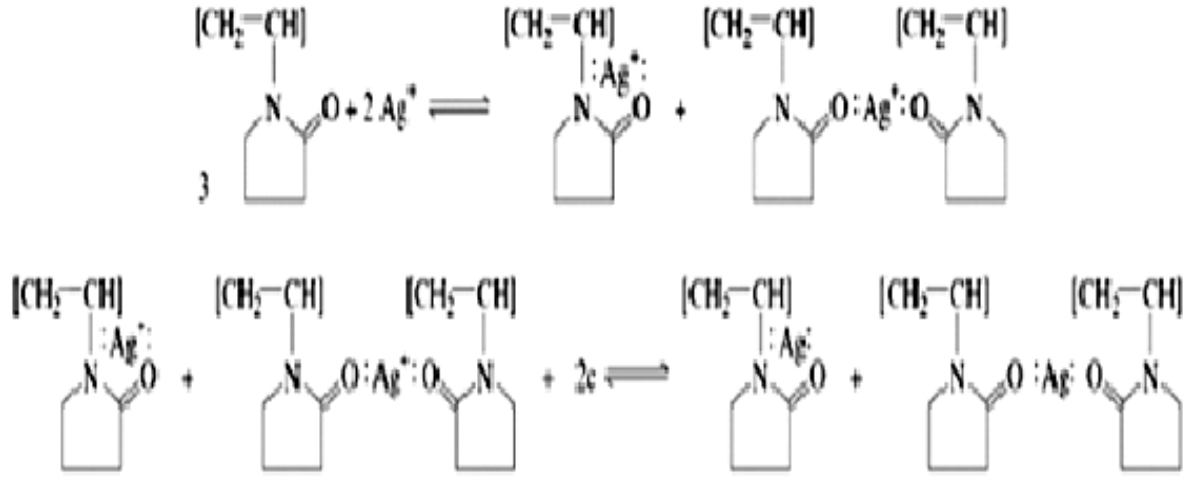

Scheme 1. Possible coordination bonding between AgNPs and PVP.

\section{Conclusion}

Radiolysis methods have proved to play an important role in the development and modification of metal nano-structures; $\gamma$-irradiation is an effective technique for preparing inorganic/organic nanocomposites. In this study, the Ag/PVP nanocomposites were synthesized using highly reducing radicals generated by steady state gamma irradiation, highly mono-dispersed. AgNPs were successively synthesized by gamma-irradiation, at different irradiation doses. UV/vis spectroscopy results showed that Ag/PVP nanocomposite exhibited a symmetric sharp absorption band peaking at the wavelength $400 \mathrm{~nm}$ confirming the presence of AgNPs. The calculated sizes of silver nano-particle using UV-VIS data are in the range 30-38 $\mathrm{nm}$. Also, TEM results have shown spherical AgNPs with narrow size distribution with a diameter of 13-30 nm depending on either irradiation dose or $\mathrm{AgNO}_{3}$ molar concentration. The XRD patterns exhibit four diffraction peaks revealed that the AgNPs are formed in the PVP matrices and their crystal structure is fcc structure. The calculated values of crystalline particle size $(\mathrm{D}, \mathrm{nm})$ showed that both $\mathrm{AgNO}_{3}$ molar concentration and irradiation dose are determining factors in controlling the particle size of AgNPs in PVP matrices. FTIR spectra illustrate 
that the formation Ag/PVP nanocomposites leads to the change in peak position, shape and intensity of some characteristic peaks. All of the differences exhibited in the Ag/PVP nanocomposite spectrum compared to the spectrum of pure PVP indicated the coordination bonding between nitrogen and AgNPs, as well as between oxygen and AgNPs, most likely through the reactions

\section{References}

Basavaraja, C., Pierson, R. and Huh, D. S. (2008) Synthesis, characterization, and comparative study of conducting polyaniline/lead titanate and polyanilinedodecylbenzenesulfonic acid/lead titanate composites. J. Appl. Polym. Sci., 108, 1070 .

Basavaraja, C., Pierson, R. and Huh, D. S. (2008) Synthesis, characterization, and comparative study of conducting polyaniline/lead titanate and polyanilinedodecylbenzenesulfonic acid/lead titanate composites. J. Appl. Polym. Sc., 108, 1070 .

Chen D. H. and Wu S. H. (2000) Synthesis of nickel nanoparticles in water-in-oil microemulsions. Chem. Mater., 12, 1354.

Evanoff, D. D. and Chumanov, G. (2004) Size-controlled synthesis of nanoparticles "Silver-only" aqueous suspensions via hydrogen reduction. The J. Physic. Chem., 108, 13948.

Jiang, P., Li, S., Xie, S., Gao, Y. and Song, L. (2004) Machinable ong PVP-stabilized silver nanowires. Chem. Eur. J., 10, 4817.

Mahendia, S., Tomar, A. K. and Kumar, S. (2011) Nano-Ag doping induced changes in optical and electrical behavior of PVA films. Mater. Sci. Engin., 176, 503.

Malinsky, M. D., Lance Kelly, K., George, C., Schatz Duyne, R. P. V. (2001) Nanosphere Lithography: Effect of substrate on the localized surface plasmon resonance spectrum of AgNPs. Phys. Chem. B., 105, 2343.

Misral, N., Biswal J., Dhamgaye, V. P., Lodha, G. S. and Sabharwal, S. (2012) A comparative study of gamma, electron beam, and synchrotron X-ray irradiation method for synthesis of AgNPs in PVP. Adv. Mater. Lett., 22, 1.

Puiso, J., Adliene, D., Guobiene, A., Prosycevas, I. and Plaipaite-Nalivaiko, R. (2011) Modification of Ag-PVP nanocomposites by gamma irradiation. Mater. Sci. Engine. B, 176, 1562.

Puiso, J., Prosycevas, I., Guobiene, A. and Tamulevicius, S. (2008) Plasmonic properties of silver in polymer. Mater. Sci. Engine., B, 149, 230.

Suyal, G. (2003) Bimetallic colloids of silver and copper in thin films: sol-gel synthesis and characterization. Thin Solid Films, 426, 53. 
Virk, H. S. and Srivastava, A. K. (2001) Modification of optical, chemical and structural response of CR-39 polymer by $50 \mathrm{Mev}$ lithium ion irradiation. Radiat. Measurem., 34, 65.

Wang, H., Qiao, X., Chen, J., Wang, X. and Ding, S. (2005) Review mechanisms of PVP in the preparation of AgNPs. Mater. Chem. Physic., 94, 449.

Wilson, R., Lynn, G., Milosavlevic, B. and Miesel, D. (2007) Within free standing PVA/PVP films. Spectrochimic aActa, Part A: Molecular and Biomolecular Spectroscopy, 95, 341.

Xie, Y. W., Ye, R. Q. and Liu, H. L. (2006) Synthesis of AgNPs in reverse micelles stabilized by natural biosurfactant. Colloid. Surf., A, 279, 175.

Zhang, Z., Zhao, B. and Hu, L. (1996) PVP protective mechanism of ultra fine silver powder synthesized by chemical reduction processes. J. Solid State Chem., 121, 105.

(Received: 01/10/2014;

accepted: 30/11/2014) 


\title{
تحضير جزيئات الفضـة النانومتريـة احاديـة التوزيـع فى وجود البولى فينيل بيروليدون باستخدام أثنعة جاما \\ زكريا اسماعيل على ، و هاى حنفى صالح ، و تامر عبد العال عفيفى

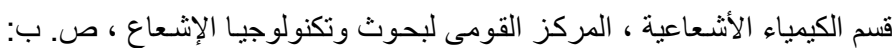

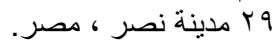

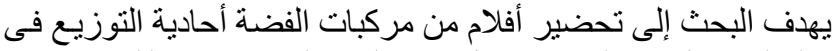

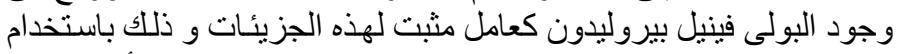

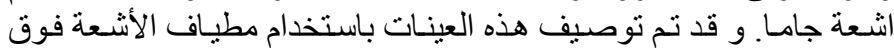

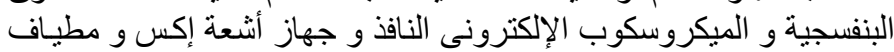

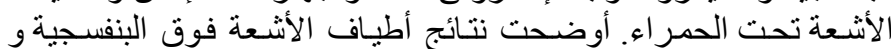

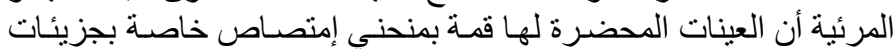

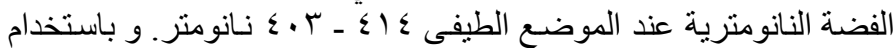

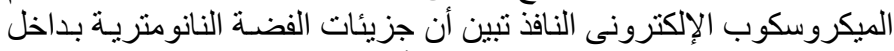

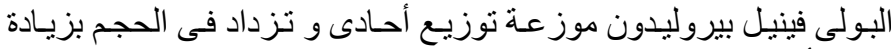
تركيز أملاح الفضة المستخدمة.

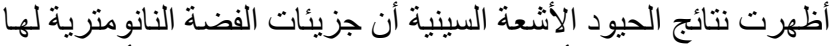

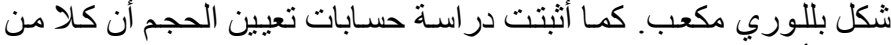

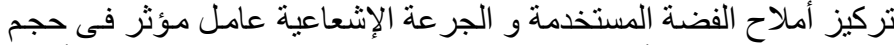

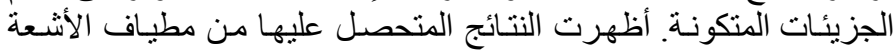

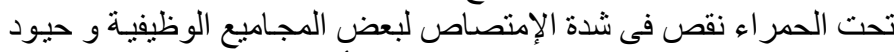

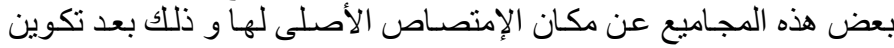

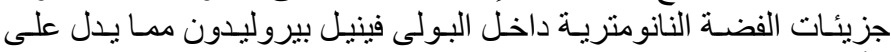

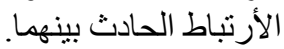

